ARTICLE

\title{
Characterization of a triad of genes in cyanophage S-2L sufficient to replace adenine by 2- aminoadenine in bacterial DNA
}

\author{
Dariusz Czernecki (i] ${ }^{1,2}$, Frédéric Bonhomme ${ }^{3}$, Pierre-Alexandre Kaminski ${ }^{4} \&$ Marc Delarue (iD ${ }^{1 凶}$
}

Cyanophage S-2L is known to profoundly alter the biophysical properties of its DNA by replacing all adenines $(A)$ with 2 -aminoadenines $(Z)$, which still pair with thymines but with a triple hydrogen bond. It was recently demonstrated that a homologue of adenylosuccinate synthetase (PurZ) and a dATP triphosphohydrolase (DatZ) are two important pieces of the metabolism of 2-aminoadenine, participating in the synthesis of ZTGC-DNA. Here, we determine that S-2L PurZ can use either AATP or ATP as a source of energy, thereby also depleting the pool of nucleotides in AATP. Furthermore, we identify a conserved gene (mazZ) located between purZ and dat $Z$ genes in $\mathrm{S}-2 \mathrm{~L}$ and related phage genomes. We show that it encodes a (d)GTP-specific diphosphohydrolase, thereby providing the substrate of PurZ in the 2-aminoadenine synthesis pathway. High-resolution crystal structures of S-2L PurZ and MazZ with their respective substrates provide a rationale for their specificities. The Z-cluster made of these three genes - datZ, mazZ and purZ - was expressed in E. coli, resulting in a successful incorporation of 2-aminoadenine in the bacterial chromosomal and plasmidic DNA. This work opens the possibility to study synthetic organisms containing ZTGC-DNA.

\footnotetext{
${ }^{1}$ Unit of Architecture and Dynamics of Biological Macromolecules, CNRS UMR 3528, 25-28 rue du Docteur Roux, Institut Pasteur, Paris, France. ${ }^{2}$ Sorbonne Université, Collège Doctoral, ED 515, Paris, France. ${ }^{3}$ Unit of Epigenetic Chemical Biology, CNRS UMR 3523, $25-28$ rue du Docteur Roux, Institut Pasteur, Paris, France. ${ }^{4}$ Unit of Biology of Pathogenic Gram-Positive Bacteria, CNRS UMR 2001, 25-28 rue du Docteur Roux, Institut Pasteur, Paris, France.

凶email: marc.delarue@pasteur.fr
} 
A t least since the last universal common ancestor (LUCA), four types of nucleobases - adenine (A), guanine (G), cytosine (C) and thymine (T) - are used to encode genetic information in the DNA of all living cells ${ }^{1}$. This principle can be extended to DNA and RNA viruses (the latter carrying uracil in lieu of thymine), which are important agents of evolution ${ }^{2}$. However, the genetic material may be subject to nucleobase modifications, for instance to confer an additional, epigenetic information or to provide resistance against restriction-modification systems. Phages T2, T4 and T6 systematically substitute 5-hydroxymethylcytosine $(5 \mathrm{hmC}$, often glucosylated) for cytosine ${ }^{3,4}$, protecting the DNA from CRISPR-Cas 9 degradation ${ }^{5}$. Likewise, phage $9 \mathrm{~g}$ replaces a quarter of its genomic guanine with archaeosine $(\mathrm{G}+)^{6}$, enabling its DNA to resist $71 \%$ of cellular restriction enzymes ${ }^{7}$.

Such alterations are made almost exclusively without changing the Watson-Crick base-pairing scheme. The only known exception to the A:T G:C pairing rule was revealed by the discovery of cyanophage S-2L, from Siphoviridae family ${ }^{8}$. S-2L abandons the usage of genomic adenine in favour of 2 -aminoadenine $(2,6$ diaminopurine or Z) (Fig. 1a). The resulting ZTGC-DNA has an improved thermal stability ${ }^{9,10}$ and proves to be almost completely resistant to adenine-targeting restriction enzymes ${ }^{11}$.

The key enzyme of $Z$ metabolism was recently identified as PurZ, a homologue of adenylosuccinate synthetases (PurA) ${ }^{12}$. PurZ of cyanophage S-2L and related vibriophage $\varphi \mathrm{VC} 8$ from Podoviridae family create the immediate precursor of 2-aminoadenine deoxyribose monophosphate (dZMP), N6-succino-2-amino-2'-deoxyadenylate monophosphate (dSMP), from standard dGMP and free aspartic acid (Asp) using ATP as the energy donor. The enzymes necessary for the subsequent conversion of dSMP to dZMP and then dZTP have not been found on the phages' genomes; instead, the non-specific enzymes of $V$. cholerae complement the pathway of dZTP metabolism of $\varphi \mathrm{VC} 8$ in vitro ${ }^{12}$.

Furthermore, in S-2L a dATP-specific triphosphatase (DatZ) eliminates dATP from the pool of available dNTPs substrates ${ }^{13}$. This step is essential for conferring an indirect nucleotide specificity to the DNA primase-polymerase (PrimPol) of the cyanophage. Synteny of both purZ and dat $Z$ genes in related viruses suggests that dATP depletion is closely tied to dZTP production.

Here, we broaden the substrate spectrum of S-2L's PurZ and present its structure in a complex with dGMP and dATP as an alternative energy donor. Moreover, along with $d a t Z$ and $p u r Z$ we identify a co-conserved gene encoding a MazG-like nucleotide phosphohydrolase (mazZ). We show that MazZ generates (d) GMP from (d)GTP, placing the enzyme directly upstream of PurZ in the 2-aminoadenine synthesis pathway. The crystal structure of MazZ bound to the intermediate product allows to identify crucial catalytic residues, shared with close homologues of the enzyme. a

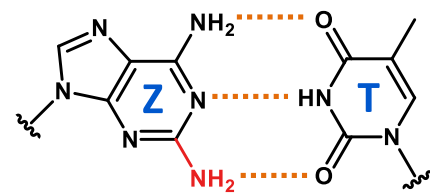

2-aminoadenine : thymine

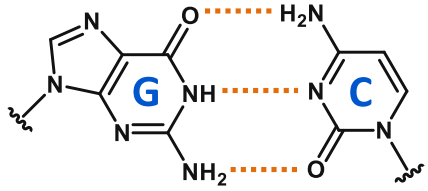

guanine : cytosine b

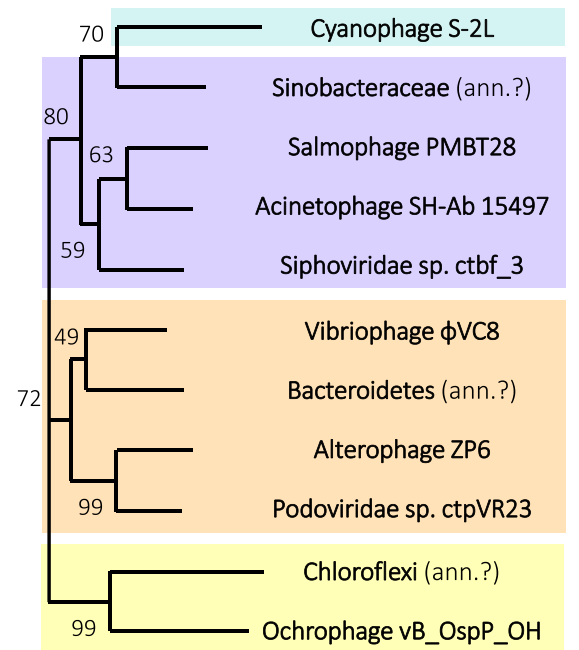

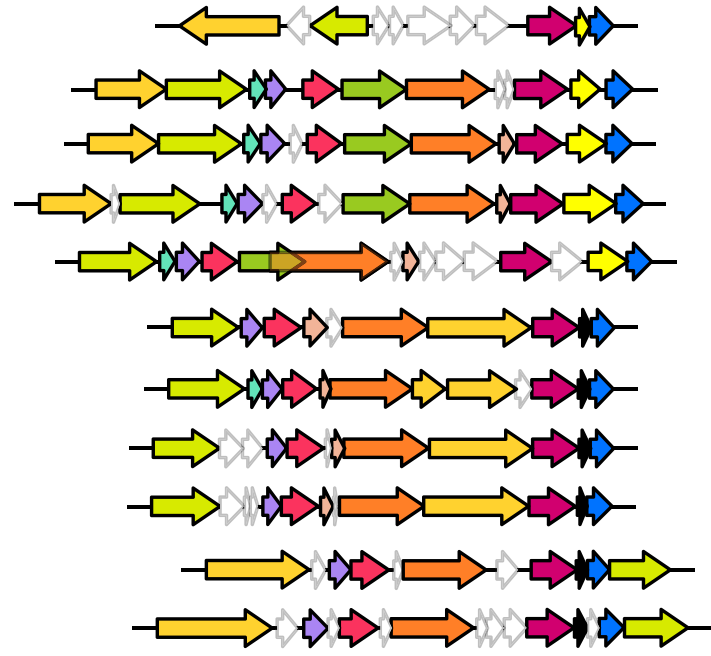

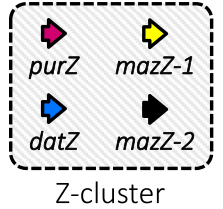

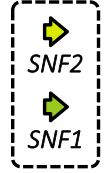

helicases

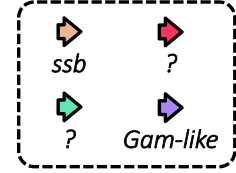

other genes

Fig. 1 ZTGC-DNA and conservation of the Z-cluster in Siphoviridae and Podoviridae phages. a Chemical structure of Z:T and G:C base pairs constituting S-2L's ZTGC-DNA. b Genomic map of most important replication genes in all phages with close purZ and datZ homologues, and their phylogeny. The phages can be presently divided into four clades (colours in background to the left) with distinct organization and variants of replication-related genes. The names of these genes, identified by their colours, are shown below. Cyanophage S-2L is, until now, the only representative of its clade. Close scrutiny of the sequence data reveals possible mis-annotation of some viral sequences with bacterial names ("ann.?" note) - their length and content perfectly match other viral sequences. 
To confirm the necessity and sufficiency of the Z-cluster (datZ, mazZ, purZ) for efficient ZTGC-DNA synthesis in cellulo, we expressed these three genes in Escherichia coli. Although toxic to the bacteria, the system was able to convert a significant amount of DNA's adenine to 2-aminoadenine both in chromosomal and plasmidic fractions.

\section{Results}

Genome of cyanophage S-2L and its relatives: a conserved Zcluster. Although the genome of phage S-2L was made available in 2004 (GenBank AX955019), homology analysis suggested several sequence errors. We decided to re-sequence it using the next-generation sequencing (NGS) technology with improved quality (GenBank MW334946; Supplementary Fig. 1). The corrected sequence is identical to the previous one with few exceptions. In particular, a mutation in the sequence between $d a t Z$ and purZ genes prevented the identification of a gene whose product corresponds to a MazG-like phosphohydrolase, named hereafter mazZ. We found no Shine-Dalgarno (SD) motifs upstream the genes, which is consistent with low SD motif conservation in cyanobacteria and their phages ${ }^{14}$. Interestingly, the S-2L genome can be divided into two parts, where almost all genes follow only one direction of translation; in-between, we identified a marRtype repressor found typically on such junctions ${ }^{15}$.

We found ten complete phage sequences having both dat $Z$ and purZ gene homologues (Fig. 1b), all coming from Spihoviridae or Podoviridae families. We inferred their phylogeny and distributed all phages into four major clusters with similar genome organization; interestingly, among them cyanophage S-2L has a unique replication module. Finally, we observed that the mazZ gene is always co-conserved between dat $Z$ and purZ, although in one of two possible variants: MazZ-1 (S-2L-like) and MazZ-2 $(\varphi \mathrm{VC} 8$-like). MazZ-1 undergoes frequent N-terminal fusions with other domains, but not in S-2L. In the following, we define the closely co-varying set of genes $\operatorname{dat} Z$, mazZ and $p u r Z$ as the Zcluster.

S-2L PurZ can function as a dATPase, with no structural selection on $2^{\prime}-\mathrm{OH}$. Structural analysis of $\varphi$ VC8 PurZ (PDB ID: $6 \mathrm{FM} 1)$ suggested no particular selection on the $2^{\prime}-\mathrm{OH}$ group of bound ATP; thus, we sought to compare the reaction time-course of PurZ with either dATP or ATP as the phosphate donor (Supplementary Fig. 2). Reactions with the purified S-2L protein followed by HPLC show no specificity of the enzyme towards the ribo and deoxy variants (Fig. 2a): it can act as a dATPase, but stays functional after dATP pool depletion by using ATP instead.

We crystallized S-2L PurZ with two of its substrates, dGMP and dATP, and solved its structure at $1.7 \AA$ resolution (Supplementary Table 1, Fig. 2b). Both ligands have a binding mode identical to the one of dGMP and ATP described previously in the structure of $\varphi \mathrm{VC} 8$ PurZ (PDB ID: 6FM1; Fig. 2c).

PurA enzymes are known to be functional dimers ${ }^{16,17}$ or even tetramers ${ }^{18}$. Through crystallographic symmetry, a PurZ dimer can be reconstructed for both S-2L and $\varphi$ VC8 (Supplementary Fig. 3A). Very low B-factors (Supplementary Fig. 3B) suggest an overall high rigidity for the dimer; the only exception is the Y272L278 loop above the reactants, in contact with the aspartate substrate $^{12}$. Its crucial tip (T273-T276) is strictly conserved between S-2L and $\varphi \mathrm{VC} 8$, both in sequence and structure.

Residues G22, S23, N49, A50, T132, Q190, V241 and the backbone atoms of Y21 form a pocket where the base moiety of dGMP is placed. Y21-S23 are positioned next to the amino group of dGMP in position 2, the hydroxyl group of the conserved S23 being $3.7 \AA$ away from the nitrogen atom. Although this distance is too long for a postulated hydrogen bond ${ }^{12}$, its polarity may still contribute to the specificity towards guanine; additionally, the partial negative charge of S23's oxygen is compatible with the close guanidino goup of R280. Residues G130, S131, R146, Y203, C204, T205 and R240 complete the dGMP pocket. In addition, V241 is ideally placed to sterically interfere with the ribose $2^{\prime}-\mathrm{OH}$ group of ribonucleotides (see below). Notably, R146 from the dimer's other molecule forms an ion pair with the $\alpha$-phosphate ${ }^{19}$.

Like ATP in $\varphi$ VC8 PurZ, the base moiety of dATP is stabilized by stacking interactions with F306 and P336 residues. Oxygen atoms from the side chains of N305, Q308 and the backbone of G335 form hydrogen bonds with adenine's 6-amino group. Importantly, the amide group of Q308 (N297 in $\varphi \mathrm{VC} 8$ ) would display conflicting polarity with the 2 -amino group of bases $G$ and $\mathrm{Z}$. The rest of the ligand interacts with S-2L PurZ almost exclusively through its triphosphate tail with residues S23, G25, G51, H52 and T53; T53 also touches the C3' atom side. The position inferred for the $2^{\prime}-\mathrm{OH}$ of ATP would be entirely exposed to the solvent, like in $\varphi \mathrm{VC} 8$ PurZ.

Molecular basis for substrate selectivity in PurZ vs PurA. To compare PurZ and PurA, we prepared a structure-informed sequence alignment. Thirty-six residues are strictly conserved between PurZ and PurA, while 24 further residues have only marginal variability (Supplementary Fig. 4). These two sets of residues cluster in the catalytic pocket and the surrounding layer, respectively (Supplementary Fig. 5A). Finally, 15 residues are unique to PurZ, but otherwise conserved in PurA. Their placement is intermediate compared to the previous classes, although several such residues (S23, T273 and Q308) interact with the substrates. In PurA, a conserved arginine (R303 in E. coli) balances the partial charge of $\mathrm{O}^{\prime}$ of IMP at $2.5-4 \AA$ and interacts with the free aspartate substrate ${ }^{20}$. The corresponding residues in PurZ extend noticeably further, being $7.9 \AA$ (S-2L R244) and $8.5 \AA$ ( $\varphi$ VC8 K267) away from $C 2{ }^{\prime}$, precluding any possible stabilization of the ribonucleotide. Moreover, in PurZ an insertion (A242-W250 for S-2L) deforms the loop that contains a conserved V241, pulling it slightly closer to the $\mathrm{C} 2$ ' ribose atomfrom 4.3 to $5.3 \AA$ as seen in PurA to $3.7 \AA$ (S-2L) and $3.6 \AA$ $(\varphi \mathrm{VC})$. Lastly, one of the two deletions specific to PurZ and PurA from Pyrococcus horikoshii ${ }^{12}$ are compensated by an additional C-terminal helix $\alpha_{11}$ unique to S-2L (Supplementary Fig. 5B). Using the above alignment, we constructed a phylogenetic tree (Supplementary Fig. 6), which confirms the possible archaeal origin for PurZ.

S-2L MazZ is a (d)GTP-specific NTP-PPase with MazG-HisE fold. Purified MazZ of cyanophage S-2L is capable of removing two terminal phosphates from a nucleoside triphosphate (Fig. 3a). Its preferential substrates, dGTP and GTP, are rapidly dephosphorylated to dGMP and GMP, respectively. In contrast, S-2L MazZ shows no substantial activity with other deoxynucleotides, including dZTP (Supplementary Fig. 7). Interestingly, dGTPspecific MazG enzymes occur in other cyanophages, that are unrelated to S-2L nonetheless ${ }^{21}$.

We obtained crystals of MazZ and solved its structure, bound to the dephosphorylation product of dGTP and catalytic $\mathrm{Mn}^{2+}$ ions, at $1.43 \AA$ resolution (Supplementary Table 1, Fig. 3b). Contrary to other members of the all- $\alpha$ NTP-PPase superfamily ${ }^{22}$, each MazZ protein chain has two additional $\beta$-strands at its C-terminus. Together, they form a homotetramer with a typical MazG-HisE fold (Supplementary Fig. 8): to our knowledge, it is the first time that the fold is recognized as identical for all MazG, MazG-like and HisE enzymes, despite the number of determined structures. The whole MazZ tetramer 

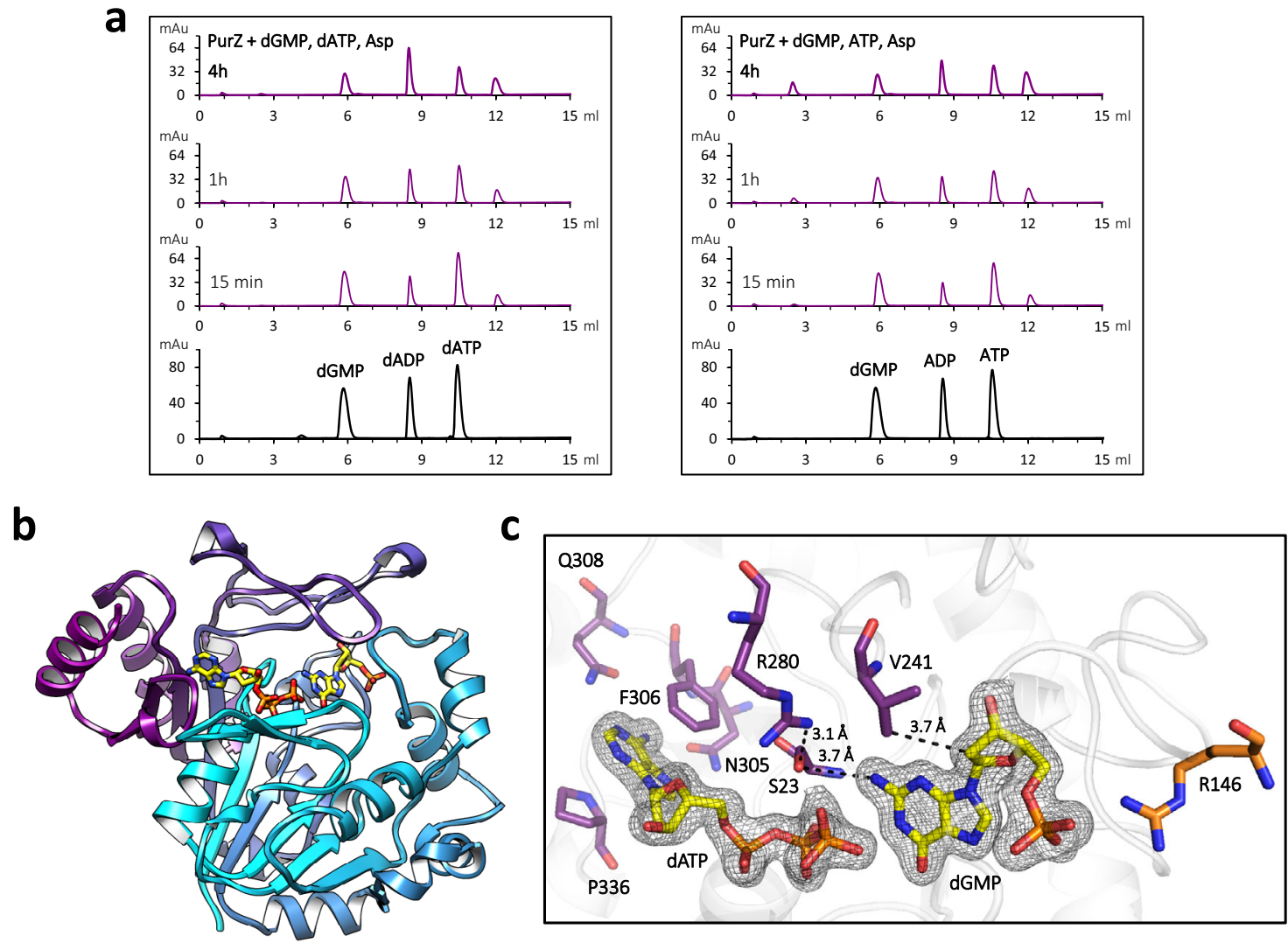

Fig. 2 dATPase activity of S-2L PurZ: functional assay and ligand-bound structure. a HPLC profiles representing the time-course of the reaction catalyzed by PurZ in presence of dGMP, Asp and dATP/ATP (purple). Pure compounds used as standards are shown below in black. The enzyme generates products corresponding to (d)ADP and dSMP. b Ribbon representation of a PurZ crystallographic protomer, with dGMP and dATP shown in stick (yellow). c Catalytic pocket of PurZ with the $2 F_{\mathrm{o}}-F_{\mathrm{c}}$ electron density contoured around the reactants at $1 \sigma$ (black mesh). Crucial residues surrounding the nucleotides are coloured by chain (purple and orange).

constitutes the asymmetric unit. The only noticeable differences in electron density between superposed chains of the tetramer lie in the solvent-exposed D43-H46 flexible loop and at both $\mathrm{N}$ - and C-termini.

The electron density of the dGTP dephosphorylation product revealed the presence of two phosphate groups (Fig. 3c). The dGDP intermediate observed in crystallo is almost completely buried inside the enzyme, except for the solvent-exposed $\beta$-phosphate next to which we found three catalytic $\mathrm{Mn}^{2+}$ ions.

From one side, the ligand is held by residues I12, W15, I16, N20, K31, E35, D53, I56, L57 and D60 of one chain. The second chain completes the pocket with residues K76, N80, W85, A92, M93, R94 and H95. The closest residue to guanine's O6 is N20, through its amide nitrogen at $3.2 \AA$; the 2 -amino group is only $3.0 \AA$ away from the carboxyl group of D60. Altogether, the specificity of MazZ emerges from the cavity volume matching guanine's shape and its charge compatibility.

We observe no steric hindrance for the possible $2^{\prime}-\mathrm{OH}$ group. Mutation of the closest I56 could affect the specificity for ribonucleotides; however, it also contacts the base's 2-amino group and is surrounded by an intricate network of other residues.

The three $\mathrm{Mn}^{2+}$ ions, named $\mathrm{A}, \mathrm{B}$ and $\mathrm{C}$, are strictly equivalent to the $\mathrm{Mg}^{2+}$ ions found in a related NTP-PPase ${ }^{23}$. Ion $\mathrm{A}$ is coordinated by residues E34, E35 and E38; ion B by E50 and D53; and ion C by E38 and E50. These ions are all coordinated in an octahedral fashion with protein, ligand and water atoms. It is possible that the two-metal-ion mechanism described for NTP-
PPases occurs in fact with two consecutive dephosphorylation steps, justifying the presence of the three ions and intermediate dGDP. In addition, residue R83, positioned only $2.8 \AA$ away from the $\beta$-phosphate, is probably important for stabilizing the transition state ${ }^{24}$.

S-2L MazZ as a representative of MazZ-1 subfamily and similarity with MazZ-2. With the exception of E34, all residues of S-2L MazZ coordinating the catalytic ions are strictly conserved across all MazZ-1 homologues (Supplementary Fig. 9). Most of the amino acids participating in nucleotide pocket formation tend to be conserved as well: only the residues I16, N20 and A92 placed around the nucleobase are unique to S-2L. Importantly, residues I56 and D60 are kept by all MazZ-1 homologues, suggesting preserved guanine specificity.

In MazZ-2, four negatively charged residues are also strictly conserved, their position corresponding to E35, E38, E50 and E53 of S-2L MazZ (Supplementary Fig. 10). However, other nucleobase-binding residues differ from MazZ-1 consensus, implying an alternative binding mode of the substrate. Finally, a counterpart of R83 important for the two-metal-ion mechanism is preserved in both variants of MazZ.

Successful conversion of $\boldsymbol{E}$. coli DNA with S-2L Z-cluster. We now propose a complete 2 -aminoadenine metabolic pathway for cyanophage S-2L and related Siphoviridae and Podoviridae phages (Fig. 4a). The post-infection composition of cellular dNTP 
a
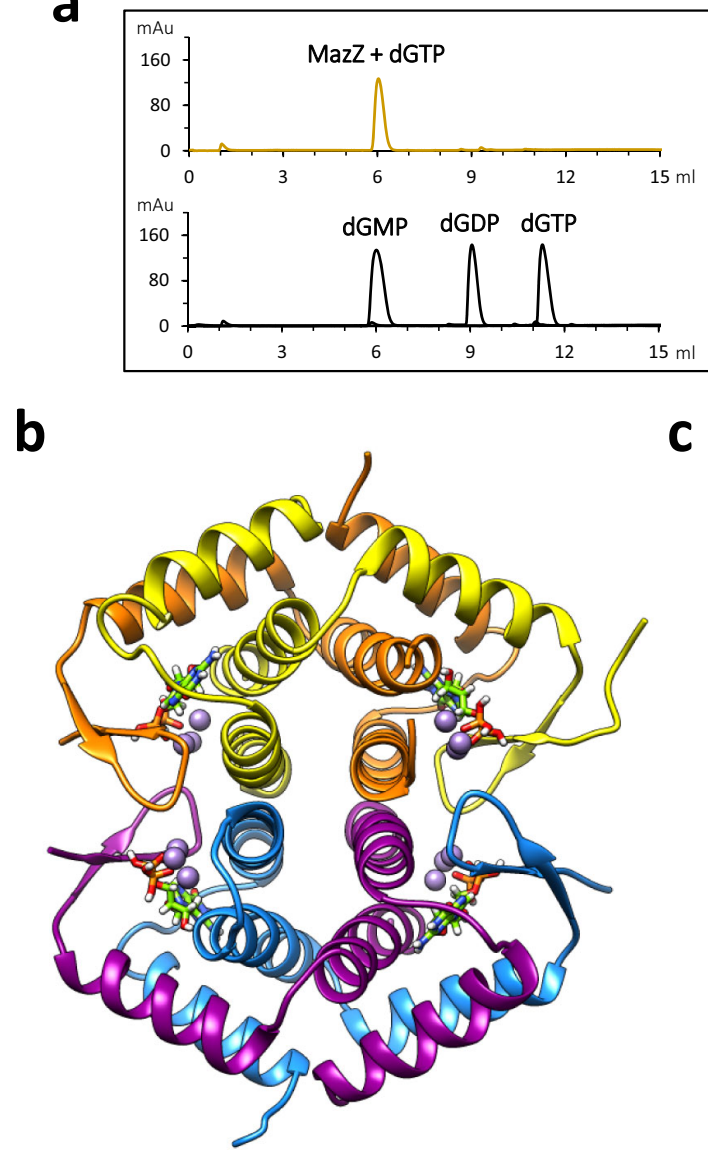

C
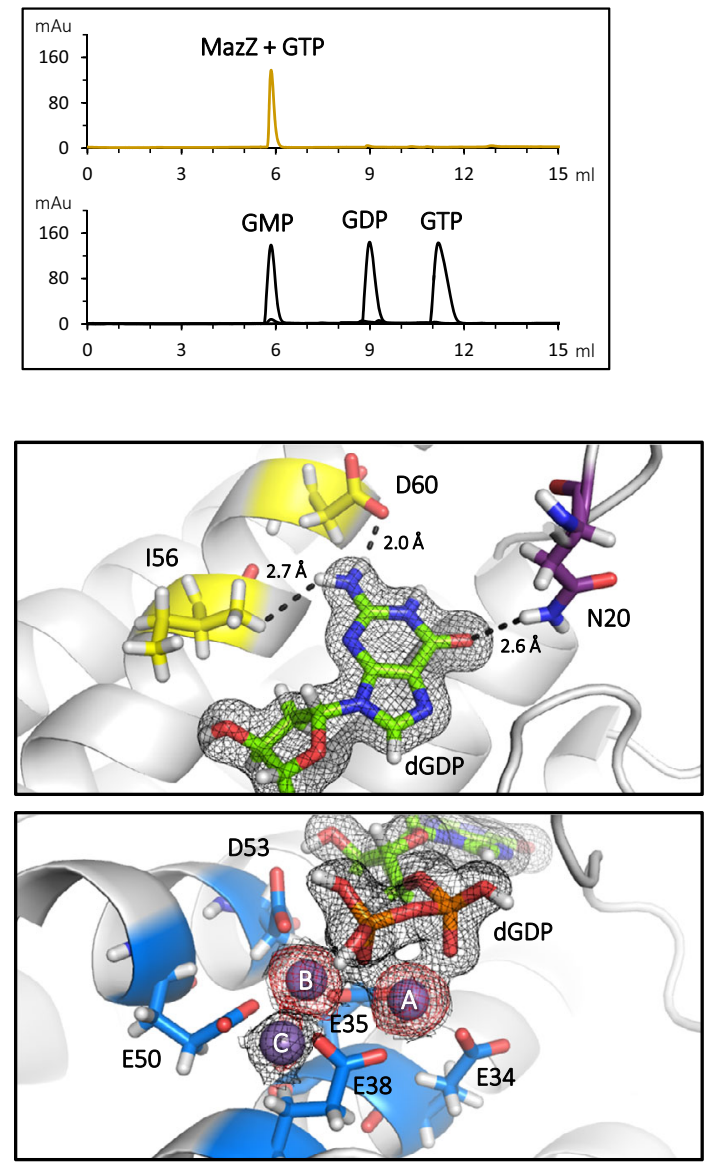

Fig. 3 Function and structure of S-2L MazZ, a (d)GTP phosphohydrolase. a HPLC analysis of S-2L MazZ activity with dGTP and GTP (gold), with nucleotide standards below (black). b A tetramer of MazZ that constitutes the crystallographic asymmetric unit. Each catalytic pocket contains the reactant (lime) and three catalytic ions and results from interactions at the interface of two chains of a tight dimer. c A close-up on the catalytic pocket, in two views. The product of dGTP dephosphorylation (lime) is identified as dGDP in the crystal, next to three catalytic $\mathrm{Mn}^{2+}$ ions (lilac spheres). The determinants of guanine specificity (yellow for N2 and purple for 06 , upper panel) and residues coordinating the ions (blue, lower panel) are placed on a single protein chain. The $2 F_{\mathrm{o}}-F_{\mathrm{c}}$ electron density around the ligands is contoured at $1 \sigma$ (black mesh); the anomalous signal attesting for the presence of $\mathrm{Mn}^{2+}$ ions is contoured at $3 \sigma$ (red mesh).

pool is heavily altered: dATP is eliminated and replaced by dZTP, which is created in turn from dGTP. In the case of cyanophage $\mathrm{S}-2 \mathrm{~L}$, the non-specific Primase-Polymerase (not conserved in other phages possessing the Z-cluster) readily inserts 2aminoadenine in front of any instructing thymine.

We put the genes dat $Z$, mazZ and purZ under the control of lac operators on compatible expression plasmids in E. coli and induced the cultures in exponential phase. The whole system proved to be toxic to the cells when expressed (Supplementary Fig. 11). Whether or not it is true for the natural hosts of S-2L and $\varphi \mathrm{VC} 8$ is uncertain, but we note that these phages are known to be lytic anyway ${ }^{8,25}$.

Using mass spectrometry, we were able to quantify the $\mathrm{Z}$ content in bacterial DNA (Fig. 4b). In plasmids and the chromosome, cells substituted on average $12.1 \%$ and $3.9 \%$ of total adenine content with 2 -aminoadenine, respectively. In addition, by quantifying the non-modified pre-induction plasmidic fraction, we normalized the plasmidic A-to-Z substitution ratio to $22.7 \%$ after expression of the Z-cluster (Fig. 4c). Importantly, incomplete Z-cluster expression yielded lower substitution rates and the effect of datZ and mazZ absence is synergistic. Altogether, it appears that bacterial DNA can be enriched with diaminopurine efficiently with the introduction of the Z-cluster alone.

\section{Discussion}

On the day of submitting this paper, we became aware of the work of Zhou et al. ${ }^{26}$ on the same subject. There, the authors identify three genes conserved in several phages that contain $\mathrm{Z}$ in their genome: purZ, also described by one of us and his collaborators $^{12}$, a gene of dATPase, which is homologous to the dat $Z$ gene that we described recently ${ }^{13}$ and a gene called DUF550, providing the dGMP substrate for the reaction catalysed by PurZ. The latter gene, described in detail for acinetophage $\mathrm{SH}-\mathrm{Ab}$ 15497 , is similar to (but longer than) the mazZ gene that we describe here in phage S-2L, and corresponds to the MazZ-2 variant as shown in Fig. 1b. In SH-Ab 15497 it is apparently both a dATPase and a dGTPase but in S-2L it is only a dGTPase, which underlines some flexibility in the strategy of these phages to incorporate $\mathrm{Z}$ instead of $\mathrm{A}$ in their genome. Another variation in this strategy comes from the DNA polymerases of ZTGC-DNA phages: all of them but S-2L have a PolA (Pol I) DNA polymerase, that was shown by Pezo et al. ${ }^{27}$ to have a definite specificity of incorporation of $Z$ vs $A$ in front of T, whereas S-2L has only a PrimPol DNA polymerase, which has no such specificity ${ }^{13}$.

Pre- and post-replicative modification pathways of genomic thymidine in bacteriophages have already been successfully transplanted to $E$. coli ${ }^{28}$ or found to be active in its lysates ${ }^{29}$. However, these changes involved groups protruding on the 
a

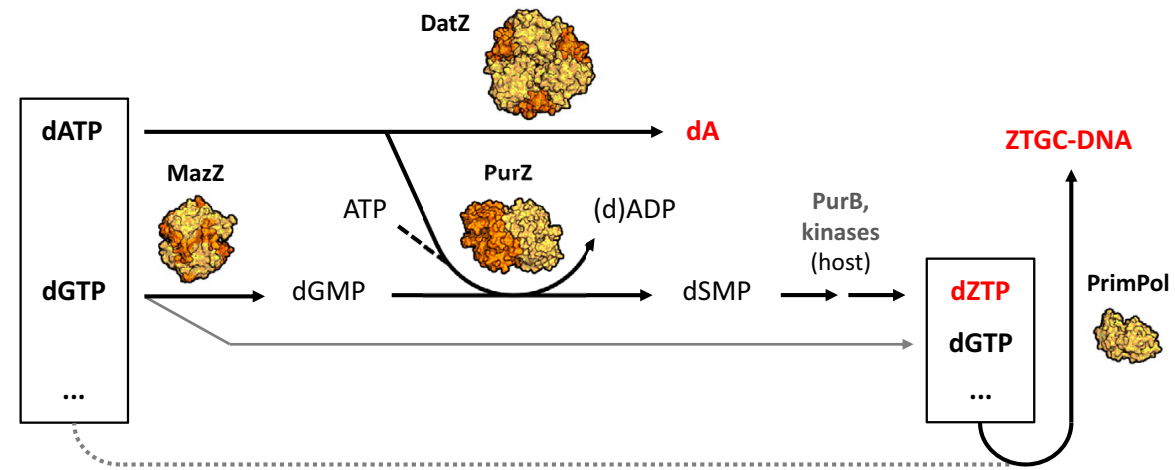

b

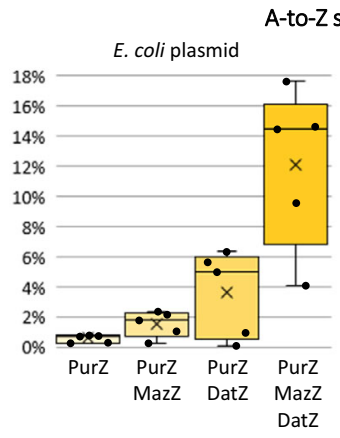

-to-Z substitution

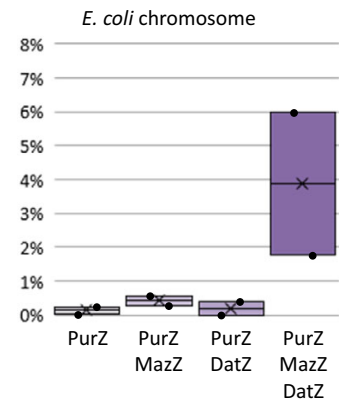

C

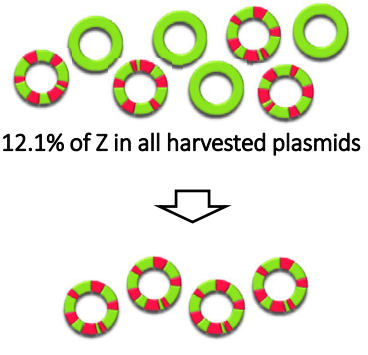

$22.7 \%$ of $Z$ in post-induction plasmids

Fig. 4 Metabolic pathway of 2-aminoadenine in S-2L phage and result of its introduction in E. coli. a Native cellular pool of nucleotides is represented in the box to the left; nucleotide pool modified through expression of the viral Z-cluster is shown on the right. Three dots represent unmodified dTTP and dCTP. Structures of S-2L proteins solved in this and previous work ${ }^{13}$ are shown next to their respective reaction arrows (to scale). Host enzymes (names in grey) finalize the dZTP pathway ${ }^{12}$. The dotted grey arrow stands for potential use of the standard dNTP pool by PrimPol in the absence of the Z-cluster. b A-to-Z substitution rates in bacterial plasmidic and chromosomal DNA, obtained after complete or partial transplantation of S-2L Z-cluster to E. coli (labels below). Box plot representation highlights data distribution, while black points denote individual expression results (pentaplicates for plasmidic DNA, duplicates for chromosomal DNA). Box whiskers delimit the range of values, box edges mark upper and lower quartiles, vertical lines represent the median, and black crosses-the average. c Accounting for the pre-induction fraction of ATGC-DNA, roughly every fourth plasmidic adenine (green) was changed to 2-aminoadenine (pink) after expressing the Z-cluster.

outside of the double helix major groove, as in epigenetic modifications. In the case of 2-aminoadenine, the additional amino group on the Watson-Crick edge profoundly modifies the stability of base pairs between the two nucleic acid strands, changing the thermodynamics of the DNA molecule rather than its external accessibility.

The kinetics of creating dZTP from dGTP has to be fine-tuned by the infecting viruses, as dGTP is also a crucial substrate for the synthesis of ZTGC-DNA. We note that in in vitro assays S-2L PurZ is overall less active than the homologous E. coli PurA (Supplementary Fig. 2), which may contribute to this effect.

In addition, we show here that the Klenow fragment of $E$. coli Pol I, a DNA polymerase participating in plasmid replication ${ }^{30}$, does not discriminate between $\mathrm{A}$ and $\mathrm{Z}$ whatsoever (Supplementary Fig. 12). This relaxed specificity holds true for most DNA and RNA polymerases ${ }^{31}$ and explains the presence of $\mathrm{Z}$ in the DNA synthesized entirely by cellular enzymes. Hosts of phages such as S-2L and $\varphi \mathrm{VC} 8$ could in principle synthesize ZTGC-DNA during the replicative stage of viral infection. However, aside from the aforementioned Z-cluster toxicity, bacterial replication may be controlled and stopped by the phages.

Lastly, the possible role of methyltransferases in the arms race of phages against bacteria can be briefly discussed: methyltransferases of $E$. coli were shown to methylate sequences containing 2-aminoadenine in vitro, although they do so with a catalytic efficiency lower by at least an order of magnitude than for regular ATGC-DNA 32,33 . In addition, phages T3 and T7 lacking the Z-cluster but belonging to the same order as $\mathrm{S}-2 \mathrm{~L}$ and $\varphi \mathrm{VC} 8$, Caudovirales, seem to undergo marginal in vivo methylation, even in mutants deprived of methylase inhibitors ${ }^{34}$. Despite the fact that 6-methyl-2-aminoadenine confers endonuclease resistance ${ }^{35}$, the effect of methylation should be small for the phages of interest or for synthetic cellular ZTGC-DNA.

The toxic effect of 2-aminoadenine in ATGC-DNA organisms such as E. coli probably stems from the complexity of the regulation of DNA transactions: sigma factors are sensitive to A-to-Z substitutions $^{36}$, while replication origins are known to require AT-rich sequences where melting is facilitated ${ }^{37}$. Nonetheless, the melting point of a $\mathrm{Z}: \mathrm{T}$ homopolymer was found to be intermediate between the A:T and G:C ones ${ }^{38}$. Attuning the cellular machinery for the presence of 2-aminoadenine should thus be in principle achievable, as it is possible for the less complex phages, potentially leading to a pure ZTGC-DNA synthetic organism.

\section{Methods}

S-2L genome sequencing, annotation and homology with other bacteriophages. S-2L DNA was isolated from phage lysate of Synechococcus elongatus culture, adapting the techniques commonly used for phage $\lambda$ DNA. The S-2L genomic library was prepared by successive DNA fragmentation, adaptor ligation, and amplification by GATC Biotech. Libraries were sequenced using Illumina HiSeq. 14,198,980 sequenced reads $(2 \times 150 \mathrm{bp})$ were obtained, covering 4,259,694,000 bases. Resulting reads were mapped against the GenBank AX955019.1 S-2L reference sequence using Minimap2 v2.1739. Variant calling was carried out by Freebayes v1.3.2 $2^{40}$ and later filtered by VCFLIB ${ }^{41}$. A consensus sequence was generated using VCF Consensus Builder v0.1.042. Annotation of the consensus sequence was carried out by translated BLAST ${ }^{43}$. Representation of the S-2L genome was made with SnapGene Viewer ${ }^{44}$. Genomic positions of genes of interest are provided in Supplementary Table 2. Nucleotide and protein sequences 
of genes purZ, mazZ, datZ, pplA, their synthetic versions and their products are specified in Supplementary Tables 3-5.

Phages related to S-2L through purZ and $\operatorname{dat} Z$ genes were found by homology searches using NCBI BLAST ${ }^{43}$, with protein sequences as separate queries. Conserved unknown genes were assessed for possible homology with known proteins using BLAST and HHpred ${ }^{45}$.

Variants MazZ-1 and MazZ-2 correspond to two sets of MazG-like family proteins whose genes are co-conserved with purZ and dat $Z$. In each set, they have an average $44 \%( \pm 6)$ and $43 \%( \pm 9)$ protein sequence identity; BLAST does not find any significant hits between the two sets. Distant identity of $31 \%$ is observed only for Ochrophage vB_OspB_OH MazZ-2 and the additional N-terminal domain of Salmophage PMBT28 fused to the C-terminal MazZ-1 sequence. Difference between MazZ-1 and MazZ-2 is further highlighted by divergent conservation profiles presented in Supplementary Data.

Protein expression and purification. Synthetic genes for expressed proteins were optimized for E. coli and synthesized using Thermo Fisher's GeneArt service. Genes were cloned into modified pRSF1-Duet expression vector with a TEV-cleavable $\mathrm{N}$ terminal 14-histidine tag ${ }^{46}$ using New England Biolabs and Anza (Thermo Fisher Scientific) enzymes. E. coli BL21-CodonPlus (DE3)-RIPL cells (Agilent) were separately transformed with engineered plasmids. Bacteria were cultivated at $37^{\circ} \mathrm{C}$ in $\mathrm{LB}$ medium with appropriate antibiotic selection (kanamycin and chloramphenicol), and induced at $\mathrm{OD}=0.6-1.0$ with $0.5 \mathrm{mM}$ IPTG. After incubation overnight at $20^{\circ} \mathrm{C}$, cells were harvested and homogenized in suspension buffer: $50 \mathrm{mM}$ Tris- $\mathrm{HCl} \mathrm{pH} 8$, $400 \mathrm{mM} \mathrm{NaCl}, 5 \mathrm{mM}$ imidazole. After sonication and centrifugation of bacterial debris, corresponding lysate supernatants were supplemented with Benzonase (SigmaAldrich) and protease inhibitor (Thermo Fisher Scientific), $1 \mu \mathrm{l}$ and 1 tablet per $50 \mathrm{ml}$, respectively. Proteins of interest were isolated by purification of the lysate on Ni-NTA column (suspension buffer as washing buffer, $500 \mathrm{mM}$ imidazole in elution buffer). Histidine tags were removed from the proteins by incubation with His-tagged TEV enzyme overnight. After removing TEV on Ni-NTA column, proteins were further purified on Superdex 200 10/300 column with $25 \mathrm{mM}$ Tris- $\mathrm{HCl} \mathrm{pH} \mathrm{8,300} \mathrm{mM} \mathrm{NaCl}$. All purification columns were from Life Sciences. Protein purity was assessed on an SDS gel (BioRad). The enzymes were concentrated to $10-19.5 \mathrm{mg} \mathrm{ml}^{-1}$ with Amicon Ultra 10k and 30k MWCO centrifugal filters (Merck), flash frozen in liquid nitrogen and stored directly at $-80^{\circ} \mathrm{C}$, with no glycerol added.

Nucleotide HPLC analysis. Thirty micromolar $\left(1.25 \mathrm{mg} \mathrm{ml}^{-1}\right)$ of S-2L PurZ or $4.2 \mu \mathrm{M}\left(0.2 \mathrm{mg} \mathrm{ml}^{-1}\right)$ of E. coli PurA was incubated at $37^{\circ} \mathrm{C}$ for $1 \mathrm{~h}$ (if not stated otherwise) with $2 \mathrm{mM}$ of respective nucleotides and $10 \mathrm{mM}$ of aspartate, in a buffer containing $50 \mathrm{mM}$ Tris pH 7.5 and $5 \mathrm{mM} \mathrm{MgSO}_{4}$. For S-2L MazZ, $10 \mu \mathrm{M}$ of the enzyme was incubated at $37^{\circ} \mathrm{C}$ for $15 \mathrm{~min}$ with $100 \mu \mathrm{M}$ of respective nucleotides, in a buffer containing $50 \mathrm{mM}$ Tris $\mathrm{pH} 7.5$ and $5 \mathrm{mM} \mathrm{MgCl}_{2}$. Reaction products were separated from the protein using 10,000 MWCO Vivaspin-500 centrifugal concentrators and stored at $-20^{\circ} \mathrm{C}$. Products and standards were assayed separately, using around $40 \mathrm{nmol}$ of each for anion-exchange HPLC on DNA-PAC100 (4x $50 \mathrm{~mm}$ ) column (Thermo Fisher Scientific). After equilibration with $150 \mu \mathrm{l}$ of a suspension buffer ( $25 \mathrm{mM}$ Tris-HCl pH $8,0.5 \%$ acetonitrile), nucleotides were injected on the column and eluted with $3 \mathrm{~min}$ of isocratic flow of the suspension buffer followed by a linear gradient of $0-200 \mathrm{mM} \mathrm{NH}_{4} \mathrm{Cl}$ over $12 \mathrm{~min}\left(1 \mathrm{ml} \mathrm{min}^{-1}\right)$. Eluted nucleotides were detected by absorbance at $260 \mathrm{~nm}$, measured in arbitrary units $[\mathrm{mAu}]$. High-purity nucleotides and chemicals were bought from SigmaAldrich, and HPLC-quality acetonitrile was from Serva.

Crystallography and structural analysis. All crystallization conditions were screened using the sitting drop technique on an automated crystallography platform ${ }^{47}$ and were reproduced manually using the hanging drop method with ratios of protein to well solution ranging from 1:2 to 2:1. PurZ was screened at $19 \mathrm{mg} \mathrm{ml}^{-1}$ with a molar excess of 1.2 of dGMP at $4{ }^{\circ} \mathrm{C}$. Capped thick rods grew over several days in 15\% v/v Tacsimate and 2\% w/v PEG 3350 buffered with $100 \mathrm{mM}$ HEPES $\mathrm{pH} 7$, and did not appear in the absence of dGMP. MazZ was screened at $14.7 \mathrm{mg} \mathrm{ml}^{-1}$ with a molar excess of 1.2 of dGTP at $18^{\circ} \mathrm{C}$. Big bundles of thin needles grew over a week in $200 \mathrm{mM} \mathrm{Li}_{2} \mathrm{SO}_{4}$ and $1.26 \mathrm{M}\left(\mathrm{NH}_{4}\right)_{2} \mathrm{SO}_{4}$ buffered with $100 \mathrm{mM}$ Tris pH 8.5. PurZ crystals were soaked for $15 \mathrm{~min}$ in a solution containing $30 \%$ glycerol, $50 \%$ dATP solution $(100 \mathrm{mM})$ and $20 \%$ crystallization buffer; MazZ crystals were soaked for several seconds with 30\% glycerol and 70\% crystallization buffer, with added $30 \mathrm{mM} \mathrm{MnCl}_{2}$. All crystals were then frozen in liquid nitrogen. Crystallographic data were collected in a nitrogen gas stream $(\sim 100 \mathrm{~K})$ at the Soleil synchrotron in France (beamlines PX1 and PX2), processed by XDS ${ }^{48}$ with the XDSME ${ }^{49}$ pipeline and refined in Phenix ${ }^{50}$. Nucleotide constraints for structure refinement were obtained using Grade Web Server ${ }^{51}$. The structure of S-2L PurZ was solved by molecular replacement with $\varphi$ VC8 PurZ model (PDB ID: 6FM1). The structure of MazZ was solved using anomalous signal from bound $\mathrm{Mn}^{2+}$ ions that guided automatic model building in Phenix AutoSol, with final manual reconstruction steps using $\operatorname{Coot}^{52}$. PurZ quaternary structure analysis was performed using PISA ${ }^{53}$ and suggested the presence of a stable dimer.

The presence of two phosphate groups in dGTP dephosphorylation product bound to MazZ was confirmed by a careful analysis of electron density and
B-factors, eliminating the possibility of a flexible $\gamma$-phosphate. Electron density corresponding to ions A, B and dGDP was also observed in a structure from a crystal not soaked in $\mathrm{MnCl}_{2}$. HPLC analysis of dGTP substrate in solution excluded dGDP contamination.

Transplantation of the Z-cluster into $E$. coli and 2-aminoadenine detection Gene dat $Z$ was cloned onto plasmid pET100/D-TOPO providing ampicillin resistance. Genes purZ and mazZ were cloned into modified pRSF1-Duet providing kanamycin resistance, in slots 1 (his-tagged) and 2, respectively, each with a ribosome-binding site upstream. Constructs with partial Z-cluster did not include mazZ in pRSF1-Duet slot 2 (datZ/purZ or purZ alone) and further had datZ replaced by mazZ on pET100/D-TOPO (mazZ/purZ). One or both plasmids were used to transform E. coli BL21-CodonPlus (DE3)-RIPL cells.

Bacteria were cultivated at $37^{\circ} \mathrm{C}$ in LB medium with appropriate antibiotic selection and induced with $1 \mathrm{mM}$ IPTG at OD $=0.60( \pm 0.03)$, or with $84 \mu \mathrm{M}$ IPTG at varying $\mathrm{OD}$ for toxicity tests. After $2 \mathrm{~h}$ of further incubation, plasmids were isolated with NucleoSpin Plasmid QuickPure kit (Macherey-Nagel) and suspended in water. When not induced, natural leaking of the promotors resulted in $0.01 \%$ of $\mathrm{Z}$ content, which was not lethal to the cells. In addition, we observed that the expression of DatZ lowers the overall plasmidic DNA yield, that can be partially compensated when both MazZ and PurZ are expressed as well; this is in line with the proposed 2-aminoadenine metabolism pathway.

Chromosomal and plasmidic DNA was digested to nucleosides with Nucleoside Digestion Mix (NEB) and separated on Amicon Ultra- $0.5 \mathrm{~mL} 10 \mathrm{~K}$ centrifugal filters. Nucleosides were analyzed by LCMS, with standard nucleoside controls (Sigma-Aldrich) or dZ (Biosynth Carbosynth). $\mathrm{dZ}$ was found to elute at the same position as dG, but with strikingly different MS/MS profiles.

The A-to-Z substitution rate was taken as a ratio of $\mathrm{dZ}$ content to $\mathrm{dZ}+\mathrm{dA}$. For plasmids, this number was further normalized to the post-induction fraction by calculating the ratio between the pre-induction and final plasmidic DNA yield.

Structure and sequence alignments, phylogeny. Structures homologous to PurZ available in PDB were identified using Dali server ${ }^{54}$. The sequences were aligned in PROMALS3D ${ }^{55}$ using structural data supplemented by full protein sequences. Graphical multialignments were prepared with ESPript $3^{56}$. PurZ/DatZ multialignment was made by aligning concatenated PurZ and DatZ sequences in the default MUSCLE algorithm in MEGA X software ${ }^{57}$. Maximum-likelihood phylogenetic trees were prepared with MEGA X with default parameters, with 100 bootstrap replications. Protein structures were analyzed with Chimera ${ }^{58}$ or Pymol $^{59}$. Distances in PurA molecules were measured using PDB IDs 1P9B, 5I34, 5K7X (Arg-O2' of IMP) and 1P9B, 2DGN, 2GCQ, 4M9D, 5I34, 5K7X (Val-C2'); for $\varphi$ VC8 PurZ measurements, PDB ID 6FKO was used.

DNA polymerase assay. Fluorescence-based polymerase activity test was executed in $20 \mathrm{mM}$ Tris- $\mathrm{HCl} \mathrm{pH} 7$ and $5 \mathrm{mM} \mathrm{MgCl}_{2}$, with $3 \mu \mathrm{M}$ of $\mathrm{dT}_{24}$ overhang DNA template, $1 \mu \mathrm{M}$ of FAM $5^{\prime}$-labelled DNA primer and various concentrations of either dATP or dZTP. The Klenow polymerase was added to final concentration of $5 \mathrm{U}$ in $50 \mu \mathrm{l}$. The assay was conducted at $37^{\circ} \mathrm{C}$ for $5 \mathrm{~min}$. Before adding the protein, DNA was hybridized by heating up to $95^{\circ} \mathrm{C}$ and gradually cooling to reaction temperature. Reactions were terminated by adding two volumes of a buffer containing $10 \mathrm{mM}$ EDTA, $98 \%$ formamide, $0.1 \%$ xylene cyanol and $0.1 \%$ bromophenol blue, and stored at $4{ }^{\circ} \mathrm{C}$. Products were preheated at $95^{\circ} \mathrm{C}$ for $10 \mathrm{~min}$, before being separated with polyacrylamide gel electrophoresis and visualized by FAM fluorescence on Typhoon FLA 9000 imager. All oligonucleotides were from Eurogentec, chemicals from Sigma-Aldrich, Klenow polymerase from Takara Bio, dATP from Fermentas (Thermo Fisher Scientific) and dZTP from TriLink BioTechnologies. Oligonucleotide sequences are specified in Supplementary Table 6.

Statistics and reproducibility. All non-crystallographic experiments were done in triplicates $(n=3)$ unless stated otherwise. For X-ray crystallography, several consistent datasets were collected from multiple crystals; the best-resolution datasets were chosen for the final refinements.

Reporting summary. Further information on research design is available in the Nature Research Reporting Summary linked to this article.

\section{Data availability}

The NGS sequence of cyanophage S-2L is deposited in the GenBank database, under the accession code MW334946. The crystallographic data for cyanophage S-2L proteins PurZ and MazZ bound to their respective ligands are deposited in the Protein Data Bank (PDB) under the accession codes 7ODX [https://doi.org/10.2210/pdb7ODX/pdb] and 7ODY [https://doi.org/10.2210/pdb7ODY/pdb]. Other data are available from the corresponding author upon request. Source data are provided with this paper.

Received: 30 April 2021; Accepted: 21 July 2021;

Published online: 05 August 2021 


\section{References}

1. Koonin, E. V. Comparative genomics, minimal gene-sets and the last universal common ancestor. Nat. Rev. Microbiol. 1, 127-136 (2003).

2. Forterre, $P$. The origin of viruses and their possible roles in major evolutionary transitions. Virus Res. 117, 5-16 (2006).

3. Wyatt, G. R. \& Cohen, S. S. The bases of the nucleic acids of some bacterial and animal viruses: the occurrence of 5-hydroxymethylcytosine. Biochem. J. 55, 774-782 (1953)

4. Lehman, I. R. \& Pratt, E. A. On the structure of the glucosylated hydroxymethylcytosine nucleotides of coliphages T2, T4, and T6. J. Biol. Chem. 235, 3254-3259 (1960).

5. Bryson, A. L. et al. Covalent modification of bacteriophage T4 DNA inhibits CRISPR-Cas9. mBio 6 (2015)

6. Thiaville, J. J. et al. Novel genomic island modifies DNA with 7-deazaguanine derivatives. PNAS 113, E1452-E1459 (2016).

7. Tsai, R., Corrêa, I. R., Xu, M. Y. \& Xu, S. Restriction and modification of deoxyarchaeosine (dG +)-containing phage 9 g DNA. Sci. Rep. 7, 8348 (2017).

8. Kirnos, M. D., Khudyakov, I. Y., Alexandrushkina, N. I. \& Vanyushin, B. F. 2Aminoadenine is an adenine substituting for a base in S-2L cyanophage DNA. Nature 270, 369 (1977).

9. Khudyakov, I. Y., Kirnos, M. D., Alexandrushkina, N. I. \& Vanyushin, B. F. Cyanophage S-2L contains DNA with 2,6-diaminopurine substituted for adenine. Virology 88, 8-18 (1978).

10. Cristofalo, M. et al. Nanomechanics of diaminopurine-substituted DNA. Biophys. J. 116, 760-771 (2019).

11. Szekeres, M. \& Matveyev, A. V. Cleavage and sequence recognition of 2,6diaminopurine-containing DNA by site-specific endonucleases. FEBS Lett. 222, 89-94 (1987).

12. Sleiman, D. et al. A third purine biosynthetic pathway encoded by aminoadenine-based viral DNA genomes. Science 372, 516-520 (2021).

13. Czernecki, D. et al. How cyanophage S-2L rejects adenine and incorporates 2aminoadenine to saturate hydrogen bonding in its DNA. Nat. Commun. 12, 2420 (2021).

14. Wei, Y. \& Xia, X. Unique Shine-Dalgarno sequences in cyanobacteria and chloroplasts reveal evolutionary differences in their translation initiation. Genome Biol. Evol. 11, 3194-3206 (2019).

15. Perera, I. C. \& Grove, A. Molecular mechanisms of ligand-mediated attenuation of DNA binding by MarR family transcriptional regulators. J. Mol. Cell Biol. 2, 243-254 (2010).

16. Wang, W., Gorrell, A., Honzatko, R. B. \& Fromm, H. J. A study of Escherichia coli adenylosuccinate synthetase association states and the interface residues of the homodimer. J. Biol. Chem. 272, 7078-7084 (1997).

17. Jayalakshmi, R., Sumathy, K. \& Balaram, H. Purification and characterization of recombinant Plasmodium falciparum adenylosuccinate synthetase expressed in Escherichia coli. Protein Expr. Purif. 25, 65-72 (2002).

18. Mehrotra, S. \& Balaram, H. Kinetic characterization of adenylosuccinate synthetase from the thermophilic archaea Methanocaldococcus jannaschii. Biochemistry 46, 12821-12832 (2007).

19. Poland, B. W. et al. Crystal structure of adenylosuccinate synthetase from Escherichia coli. Evidence for convergent evolution of GTP-binding domains. J. Biol. Chem. 268, 25334-25342 (1993).

20. Wang, W., Poland, B. W., Honzatko, R. B. \& Fromm, H. J. Identification of arginine residues in the putative L-aspartate binding site of Escherichia coli adenylosuccinate synthetase. J. Biol. Chem. 270, 13160-13163 (1995).

21. Rihtman, B. et al. Cyanophage MazG is a pyrophosphohydrolase but unable to hydrolyse magic spot nucleotides. Environ. Microbiol. Rep. 11, 448-455 (2019).

22. Moroz, O. V. et al. Dimeric dUTPases, HisE, and MazG belong to a new superfamily of all- $\alpha$ NTP pyrophosphohydrolases with potential "housecleaning" functions. J. Mol. Biol. 347, 243-255 (2005).

23. Moroz, O. V. et al. The crystal structure of a complex of Campylobacter jejuni dUTPase with substrate analogue sheds light on the mechanism and suggests the "basic module" for dimeric d(C/U)TPases. J. Mol. Biol. 342, 1583-1597 (2004).

24. Kim, E. E. \& Wyckoff, H. W. Reaction mechanism of alkaline phosphatase based on crystal structures: two-metal ion catalysis. J. Mol. Biol. 218, 449-464 (1991).

25. Solís-Sánchez, A. et al. Genetic characterization of ØVC8 lytic phage for Vibrio cholerae O1. Virol. J. 13, 47 (2016).

26. Zhou, Y. et al. A widespread pathway for substitution of adenine by diaminopurine in phage genomes. Science 372, 512-516 (2021).

27. Pezo, V. et al. Noncanonical DNA polymerization by aminoadenine-based siphoviruses. Science 372, 520-524 (2021).

28. Mehta, A. P. et al. Replacement of thymidine by a modified base in the Escherichia coli genome. J. Am. Chem. Soc. 138, 7272-7275 (2016).

29. Lee, Y.-J. et al. Identification and biosynthesis of thymidine hypermodifications in the genomic DNA of widespread bacterial viruses. PNAS 115, E3116-E3125 (2018).

30. Camps, M. \& Loeb, L. A. When Pol I goes into high gear: processive DNA synthesis by Pol I in the cell. Cell Cycle 3, 114-116 (2004).
31. Bailly, C. \& Waring, M. J. The use of diaminopurine to investigate structura properties of nucleic acids and molecular recognition between ligands and DNA. Nucleic Acids Res. 26, 4309-4314 (1998).

32. Marzabal, S. et al. Dam methylase from Escherichia coli: kinetic studies using modified DNA oligomers: hemimethylated substrates. Nucleic Acids Res. 23, 3648-3655 (1995)

33. Brennan, C. A., Cleve, M. D. V. \& Gumport, R. I. The effects of base analogue substitutions on the methylation by the EcoRI modification methylase of octadeoxyribonucleotides containing modified EcoRI recognition sequences. $J$. Biol. Chem. 261, 7279-7286 (1986).

34. Krüger, D. H. et al. DNA methylation of bacterial viruses T3 and T7 by different DNA methylases in Escherichia coli K12 cells. Eur. J. Biochem. 150 323-330 (1985).

35. Brennan, C. A., Van Cleve, M. D. \& Gumport, R. I. The effects of base analogue substitutions on the cleavage by the EcoRI restriction endonuclease of octadeoxyribonucleotides containing modified EcoRI recognition sequences. J. Biol. Chem. 261, 7270-7278 (1986).

36. Feklistov, A. \& Darst, S. A. Structural basis for promoter -10 element recognition by the bacterial RNA polymerase $\sigma$ subunit. Cell 147, 1257-1269 (2011).

37. Rajewska, M., Wegrzyn, K. \& Konieczny, I. AT-rich region and repeated sequences - the essential elements of replication origins of bacterial replicons. FEMS Microbiol. Rev. 36, 408-434 (2012).

38. Sági, J., Szakonyi, E., Vorlíčková, M. \& Kypr, J. Unusual contribution of 2 aminoadenine to the thermostability of DNA. J. Biomol Struct. Dyn. 13, 1035-1041 (1996).

39. Li, H. Minimap2: pairwise alignment for nucleotide sequences. Bioinformatics 34, 3094-3100 (2018)

40. Garrison, E. \& Marth, G. Haplotype-based variant detection from short-read sequencing. Preprint at https://arxiv.org/abs/1207.3907 (2012).

41. Garrison, E., Kronenberg, Z. N., Dawson, E. T., Pedersen, B. S. \& Prins, P. $\mathrm{Vcflib}$ and tools for processing the VCF variant call format. Preprint at bioRxiv https://doi.org/10.1101/2021.05.21.445151 (2021).

42. Kruczkiewicz, P. VCF Consensus Builder (Python Package Index repository, 2019)

43. Wheeler, D. L. et al. Database resources of the National Center for Biotechnology. Nucleic Acids Res. 31, 28-33 (2003).

44. SnapGene software (Insightful Science; available at snapgene.com, 2021).

45. Zimmermann, L. et al. A completely reimplemented MPI bioinformatics toolkit with a new hhpred server at its core. J. Mol. Biol. 430, 2237-2243 (2018).

46. Sauguet, L., Raia, P., Henneke, G. \& Delarue, M. Shared active site architecture between archaeal PolD and multi-subunit RNA polymerases revealed by X-ray crystallography. Nat. Commun. 7, 12227 (2016).

47. Weber, P. et al. High-throughput crystallization pipeline at the crystallography core facility of the Institut Pasteur. Molecules 24, 4451 (2019).

48. Kabsch, W. XDS. Acta Cryst. D. 66, 125-132 (2010)

49. Legrand, P. XDS Made Easier (GitHub repository, 2017).

50. Liebschner, D. et al. Macromolecular structure determination using X-rays, neutrons and electrons: recent developments in Phenix. Acta Cryst. D. 75, 861-877 (2019).

51. Bricogne, G. et al. BUSTER version 1.2.13. Global Phasing Ltd (2017)

52. Emsley, P., Lohkamp, B., Scott, W. G. \& Cowtan, K. Features and development of Coot. Acta Cryst. D. 66, 486-501 (2010).

53. Krissinel, E. \& Henrick, K. Inference of macromolecular assemblies from crystalline state. J. Mol. Biol. 372, 774-797 (2007).

54. Holm, L. Benchmarking fold detection by DaliLite v.5. Bioinformatics. https:// doi.org/10.1093/bioinformatics/btz536. (2019).

55. Pei, J., Kim, B.-H. \& Grishin, N. V. PROMALS3D: a tool for multiple protein sequence and structure alignments. Nucleic Acids Res. 36, 2295-2300 (2008)

56. Robert, X. \& Gouet, P. Deciphering key features in protein structures with the new ENDscript server. Nucleic Acids Res. 42, W320-W324 (2014).

57. Kumar, S., Stecher, G., Li, M., Knyaz, C. \& Tamura, K. MEGA X: molecular evolutionary genetics analysis across computing platforms. Mol. Biol. Evol. 35, 1547-1549 (2018)

58. Pettersen, E. F. et al. UCSF Chimera-a visualization system for exploratory research and analysis. J. Computat Chem. 25, 1605-1612 (2004).

59. The PyMOL Molecular Graphics System, Version 1.8 Schrödinger, LLC (2015)

\section{Acknowledgements}

We thank the Crystallogenesis and Crystallography Platform (PF6) of Institut Pasteur for help in crystallization and preliminary crystallographic data collection. We thank Marce Hollenstein and his group for the use of their HPLC system. We also thank staff from PROXIMA-1 and PROXIMA-2 beamlines for help in data collection and SOLEIL (Saint-Aubin, France) and for provision of synchrotron radiation facilities. We thank Pierre Legrand for discussions regarding the structural aspect of this work, as well as Laurent Debarbieux and his group for discussions regarding phages. We also thank 
Stéphane Decorps-Declere from the Hub de Bioinformatique et Biostatistique (C3BI, Institut Pasteur) for help with the S-2L genome mapping and its deposition. The authors acknowledge a DIM1Health 2019 grant from the Région Ile de France to the project EpiK (coordinated by P.B. Arimondo) for the LCMS equipment.

\section{Author contributions}

M.D. directed the study. D.C. and M.D. designed the study. D.C., F.B. and P.-A.K. performed experiments. D.C., F.B., P.-A.K. and M.D. analyzed the data. D.C. and M.D. wrote the manuscript.

\section{Competing interests}

The work on the possibility to use this triad of genes to put the 2-aminoadenine base in the genome of a bacterium is the subject of a U.S. provisional patent application No. 63/ 172.070 filed on April 07, 2021 entitled "2-AMINOADENINE MODIFIED NUCLEIC ACIDS, CELLS COMPRISING THEM, AND METHODS OF PRODUCING THEM” by D.C., P.-A.K and M.D. F.B. declares no competing interest.

\section{Additional information}

Supplementary information The online version contains supplementary material available at https://doi.org/10.1038/s41467-021-25064-x.

Correspondence and requests for materials should be addressed to M.D.
Peer review information Nature Communications thanks Mariusz Jaskolski and Shuangyong $\mathrm{Xu}$ for their contribution to the peer review of this work.

Reprints and permission information is available at http://www.nature.com/reprints

Publisher's note Springer Nature remains neutral with regard to jurisdictional claims in published maps and institutional affiliations.

(c) Open Access This article is licensed under a Creative Commons Attribution 4.0 International License, which permits use, sharing, adaptation, distribution and reproduction in any medium or format, as long as you give appropriate credit to the original author(s) and the source, provide a link to the Creative Commons license, and indicate if changes were made. The images or other third party material in this article are included in the article's Creative Commons license, unless indicated otherwise in a credit line to the material. If material is not included in the article's Creative Commons license and your intended use is not permitted by statutory regulation or exceeds the permitted use, you will need to obtain permission directly from the copyright holder. To view a copy of this license, visit http://creativecommons.org/ licenses/by/4.0/.

(C) The Author(s) 2021 